\title{
"I don’t need supporting, me, I just do it." A qualitative study of the social influences in male weight loss behaviours
}

\author{
K.A. Harcourt ${ }^{1}$, M.E. Clegg ${ }^{1}$, J.V. Appleton ${ }^{2}$ and L. Hunter ${ }^{2}$ \\ ${ }^{1}$ Department of Sport and Health Sciences, Oxford Brookes University, Oxford, OX3 OBP, UK and \\ ${ }^{2}$ Department of Social Work and Public Health, Oxford Brookes University, Oxford, OX3 OFL, UK
}

Data from the 2015 Health Survey for England (HSE) shows that between 1993 and 2015, obesity in men rose from $13.2 \%$ to $27.0 \%$, and the proportion of men with a healthy weight BMI decreased from $41 \%$ to $30 \%{ }^{(1)}$. The development of overweight and obesity is associated with food and exercise behaviours ${ }^{(2)}$. Social influences, such as family and friends, may influence dietary, exercise and weight loss behaviours ${ }^{(3,4)}$. Hence the aim of the current study was to explore the social influences in men's accounts of their dietary, physical activity and weight loss behaviours.

Semi-structured interviews were conducted ( $n$ 19) with men with a Body Mass Index (BMI) between $24-40 \mathrm{~kg} / \mathrm{m}^{2}$, aged $18-60$ years living in one county in the South West of the UK. Participants were healthy, not dieting or usually active. Participants took part in one interview with the researcher and completed a short socio-economic status questionnaire at the time of the interview. Ethical approval was obtained from Oxford Brookes University. Interviews were audio recorded, transcribed verbatim and coded line-by-line using Thematic Analysis ${ }^{(5)}$ and NVivo 11 software.

$\begin{array}{ll}\text { Themes } & \\ \text { The Influence of Family } & \text { Interactions with Professionals } \\ \text { The Influence of Partners } & \text { Society and Culture } \\ \text { The Influence of Friends and Peers } & \text { Weight Loss Groups }\end{array}$

Six themes were inductively derived from the data; The Influence of Family, The Influence of Partners, The Influence of Friends and Peers, Interactions with Professionals, Society and Culture, and Weight Loss Groups. Among the men that were fathers, their food and physical activity decisions were influenced by concerns for their children's health as well as their own. Fathers were responsible for their child's food education, not food intake. Engaging in active play with their children was a key construct in being a good father. Partners were included in goal-sharing and decision making regarding food and weight loss. They heavily influenced the meals that were eaten within the home environment. Partners primarily promoted weight loss diets rather than physical activity, however, some men did not feel they needed any weight loss support at all. Men talked to their friends about exercise and food intake in general, but not for "support". Many discussed their doctor as a source of support and information. Men of all ages discussed health and weight concerns, with several voicing body image dissatisfaction. To gain a deeper insight into the role of family members on male weight loss behaviours, future research could include interviews with men's partners and children.

1. Moody A \& Neave A (2016) Health Survey for England 2015.

2. Shaw K, Gennat H, O'Rourke P et al. (2006) Cochrane Database Syst Rev.

3. Maclean A, Hunt K, Gray C, Smillie S et al. (2014) Int J Mens Health 13, 121-138.

4. De Souza P \& Ciclitira KE (2005) J Health Psychol 10, 793-804.

5. Braun V \& Clarke V (2006) Qual Res Psychol 3, 77-110. 